\title{
A Parallel Algorithm for Partitioning a Point Set to Minimize the Maximum of Diameters
}

\author{
Muhammad H. Alsuwaiyel \\ Department of Information and Computer Science \\ King Fahd University of Petroleum \& Minerals \\ Dhahran 31261, Saudi Arabia \\ e-mail:suwaiyel@ccse.kfupm.edu.sa
}

\begin{abstract}
Given a set $S$ of $n$ points in the plane, we consider the problem of partitioning $S$ into two subsets such that the maximum of their diameters is minimized. We present a parallel algorithm to solve this problem that runs in time $O(\log n)$ using the CREW PRAM with $O\left(n^{2}\right)$ processors.
\end{abstract}

\section{Introduction}

Let $S$ be a set of $n$ points in the plane. The problems of partitioning $S$ into $k$ partitions, or covering $S$ with $k$ convex objects, e.g. disks, are intractable. When the number of partitions or convex objects is restricted to two, a number of algorithms have been developed in order to meet a given criterion. For the case when the problem is partitioning $S$ into two partitions $S_{1}$ and $S_{2}$ and the criterion is to minimize the maximum of the two diameters, Avis [3] gave a sequential algorithm to solve this problem in $O\left(n^{2} \log ^{2} n\right)$ time and $O\left(n^{2}\right)$ space. Later, Asano et al [2] improved the bound to $O(n \log n)$ time and $O(n)$ space. Another $O(n \log n)$ time algorithm that is simpler was given by Monma and Suri [4]. Other parallel algorithm for this problem were not found in the literature.

In this paper, we present a special purpose parallel algorithm to solve this problem that runs in time $O(\log n)$ using the CREW PRAM model of computation with $O\left(n^{2}\right)$ processors. 


\section{The structure of an optimal bipartition}

For simplicity, we will assume that each point has only one farthest neighbor. Modifying the algorithm to the more general case where a point may have more than one farthest neighbor is easy. If $p$ and $q$ are two points in $S$, we will denote by $d(p, q)$ the Euclidean distance between $p$ and $q$. For $A \subseteq S$, we define $\operatorname{Diam}(A)$ to be the diameter of $A$, that is, the largest distance realized by two points in $A$. A bipartition $\{A, B\}$ will be called optimal if the maximum of the two diameters of $A$ and $B$ is minimum among all bipartitions of $S$. For each point $p$ in $S$, let $f(p)$ denote the farthest neighbor of $p$. In general, we define $f^{0}(p)=p$, and $f^{j}(p)=f\left(f^{j-1}(p)\right)$.

Definition 1 Let $C \subset S$ and $C^{\prime} \subseteq S-C$ such that for all $p \in C$ and all $q \in C^{\prime}, f(p) \in C^{\prime}$ and $f(q) \in C$. Then $C$ and $C^{\prime}$ will be called clusters, and the pair $\left(C, C^{\prime}\right)$ a cluster pair.

The following lemma provides the basis for the algorithm to be developed.

Lemma 1 Let $\{A, B\}$ be a bipartition of the set of points in $S$, and $p \in A$. If $f(p) \in A$, then $\max \{\operatorname{Diam}(A-\{p\}), \operatorname{Diam}(B \cup\{p\})\} \leq \max \{\operatorname{Diam}(A), \operatorname{Diam}(B)\}$.

proof. Let $D=\max \{\operatorname{Diam}(A), \operatorname{Diam}(B)\}$, and suppose that both $p$ and $f(p)$ are in $A$. If for all $x \in S, d(p, x)<D$, then moving $p$ to $B$ will not increase the maximum of diameters. On the other hand, if for some $x \in S, d(p, x)=D$, then since $D=d(p, x) \leq d(p, f(p))$, moving $p$ to $B$ will not increase the maximum of diameters. It follows that in both cases

$$
\max \{\operatorname{Diam}(\{A-\{p\}), \operatorname{Diam}(B \cup\{p\})\} \leq D
$$

Corollary 1 There is an optimal bipartition $\{A, B\}$ such that for all cluster pairs $\left(C, C^{\prime}\right)$, either $C \subseteq A$ and $C^{\prime} \subseteq B$ or vise-versa.

The function $f$ defined above has the property that if $q=f(p), w=f(q)$ and $p \neq w$, then $d(p, q)<d(q, w)$. In general, for any $j \geq 1$ and any point $p \in S$, if $f^{j-1}(p) \neq f^{j+1}(p)$, then

$$
d\left(f^{j-1}(p), f^{j}(p)\right)<d\left(f^{j}(p), f^{j+1}(p)\right)
$$




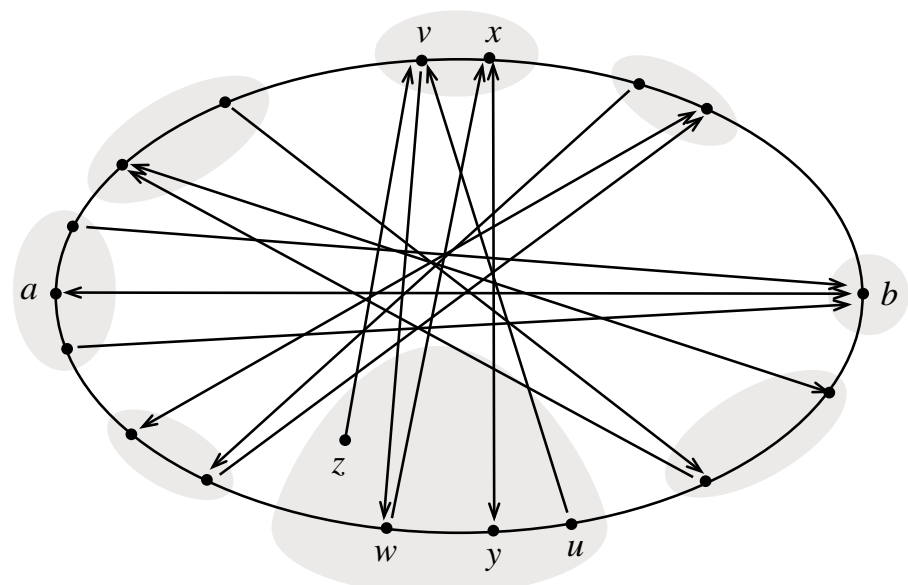

Figure 1: Partitioning the points into clusters.

For example, in Figure 1,

$$
d(u, v)<d(v, w)<d(w, x)<d(x, y)=d(y, x)
$$

In this figure, $f(p)=q$ is represented by an arrow directed from $p$ to $q . \quad f(p)=q$ and $f(q)=p$ is represented by a line segment with arrows at both ends. For clarity, only one point not on the convex hull is shown.

Thus, $f$ induces a directed graph $G=(S, E)$ with the property that the only cycles in $G$ are of length 2 , and they are pairs of points $(p, q)$ such that $f(p)=q$ and $f(q)=p$. Since, by assumption, $f$ is a function (each point has exactly one farthest neighbor), it follows that if $G$ contains more than one cycle, then it is disconnected. By Inequality 1 and the definition of a cluster pair, each cluster pair $\left(C, C^{\prime}\right)$ contains exactly two points $p \in C_{1}$ and $q \in C_{2}$ such that $f(p)=q$ and $f(q)=p$. It follows that each component of the directed graph corresponds to exactly one cluster pair. Figure 2 shows the component of the directed graph $G$ corresponding to the cluster pair $(\{v, x\},\{z, w, y, u\})$ shown in Figure 1. Note that all points in the interior of the convex hull are leaves in their respective components.

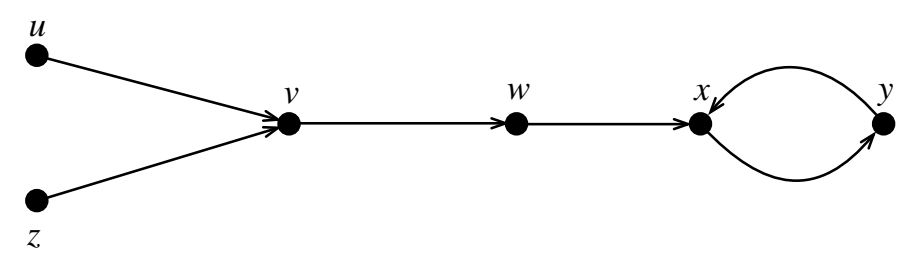

Figure 2: One component of the graph. 
For any component $H$ of the graph $G$ corresponding to a cluster pair $\left(C, C^{\prime}\right)$, let $r_{1}$ and $r_{2}$ denote the two points constituting the cycle in $H$. Define the function $g(p)=f^{2 n}(p)$. Since there is only one cycle in $H$ whose vertices, namely $r_{1}$ and $r_{2}$, are reachable from all other points in $H, g(p)$ is either $r_{1}$ or $r_{2}$. As $g(p)$ is within even edge distance from $p, g(p)$ is in the same cluster as $p$. Consequently, the function $g$ induces two shallow directed trees rooted at $r_{1}$ and $r_{2}$. Each tree consists of a root $r$ and a number of children that point to it, namely those points within even distance from $r$. Thus, each directed tree represents exactly one cluster, and each cluster is represented by a directed tree. As an example, the component shown in Figure 2 is transformed by the function $g$ into the two directed trees shown in Figure 3. It follows that $H$ is transformed by $g$ into two directed trees corresponding to $C$ and $C^{\prime}$. It should be noted that both the directed graph defined by $f$ and the directed trees defined by $g$ are represented by the algorithm using two arrays of size $n$.
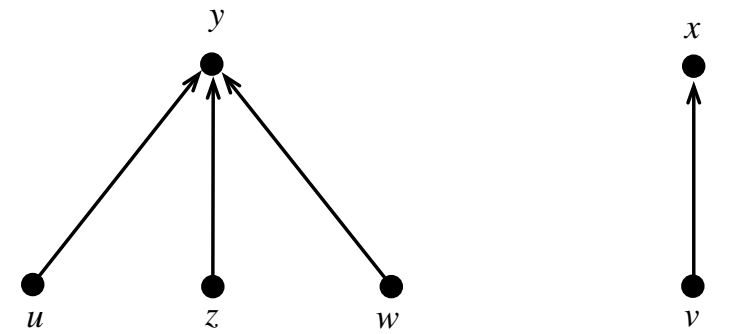

Figure 3: Two trees corresponding to two clusters in a cluster pair.

Let $P$ be the polygon formed by the points on $C H(S)$, the convex hull of $S$. It is well-known that for all $p \in S, f(p)$ is a vertex of $P$. Let $k$ be the number of cluster pairs, and $C_{0}$ any cluster. For $1 \leq j \leq 2 k-1$, let $C_{j}$ be such that $C_{j} \cap C H(S)$ follows $C_{j-1} \cap C H(S)$ in a clockwise traversal of the boundary of $P$. It is fairly easy to show that for $0 \leq j \leq k-1$, $\left(C_{j}, C_{j+k}\right)$ is a cluster pair. By Corollary 1 , we may assume that $C_{j}$ belongs to one partition, and $C_{j+k}$ belongs to the other. Clearly, each partition must consist of exactly $k$ adjacent clusters, that is, their intersection with $C H(S)$ consists of one polygonal chain. As a result, there are only $k$ possibilities for bipartitioning $S$ with the property that the two clusters in a cluster pair are assigned to different partitions. By exhaustively computing the $k$ maximums of diameters, the partitioning that results in the minimum of these maximums is selected. 


\section{The partitioning algorithm}

The partitioning algorithm can now be described as follows.

1. Compute $C H(S)$, the convex hull of $S$, and let $h=|C H(S)|$. This takes $O(\log n)$ time using $O(n)$ processors [1].

2. For each point $p \in S$, allocate $\lceil h / \log h\rceil$ processors. Since $f(p) \in C H(S)$, these processors will compute $f(p)$ in $O(\log h)$ time.

3. In this step we construct the function $g$ using pointer jumping in which each processor associated with point $p$ executes the assignment $f(p)=f(f(p))$ repeatedly until $f(f(f(p)))=f(p)$. The number of applications of the function $f$ is $O(\log n)$, as the size of each component is $O(n)$ and the unique cycle it contains is of length 2. Hence, at the end of this step, we will have computed the $2 k$ clusters as directed trees. We number them clockwise around the boundary of $C H(S)$ as $C_{0}, C_{1}, \ldots, C_{2 k-1}$, where $C_{0}$ is chosen arbitrarily. Finally, we assign label $j$ to every point in cluster $C_{j}$.

4. Label $k n$ processors as $P_{j, i}, 0 \leq j \leq k-1,1 \leq i \leq n$.

For $j=0,1, \ldots, k-1$ do in parallel: Processors $P_{j, i}, 1 \leq i \leq n$, partition the point set into $S_{j}$ and $S_{j}^{\prime}$, where $S_{j}$ is the set of points whose label is in $\{j, j+1, \ldots, j+k-1\}$, and $S_{j}^{\prime}$ is the set of points whose label is in $\{j+k, j+k+1, \ldots, j-1\}$. Compute $\operatorname{Diam}\left(S_{j}\right)$ and $\operatorname{Diam}\left(S_{j}^{\prime}\right)$. Computing the two diameters using $n$ processors takes $O(\log n)$ time [1]. Thus, the total time required in this step is $O(\log n)$ using $k n=$ $O(h n)$ processors, as $h$ is an upper bound on the number of clusters.

5. Among the pairs $\left(D_{0}, D_{0}^{\prime}\right),\left(D_{1}, D_{1}^{\prime}\right), \ldots,\left(D_{k-1}, D_{k-1}^{\prime}\right)$, Return that pair $\left(D_{j}, D_{j}^{\prime}\right)$ in which $\max \left\{D_{j}, D_{j}^{\prime}\right\}$ is minimum, and the corresponding partitions.

Figure 4 shows an example in which the set of points is partitioned into 8 clusters. In this example the following 8 diameters are computed in parallel:

$$
\begin{array}{ll}
D_{0}=\operatorname{Diam}\left(C_{0} \cup C_{1} \cup C_{2} \cup C_{3}\right) & D_{0}^{\prime}=\operatorname{Diam}\left(C_{4} \cup C_{5} \cup C_{6} \cup C_{7}\right) \\
D_{1}=\operatorname{Diam}\left(C_{1} \cup C_{2} \cup C_{3} \cup C_{4}\right) & D_{1}^{\prime}=\operatorname{Diam}\left(C_{5} \cup C_{6} \cup C_{7} \cup C_{0}\right)
\end{array}
$$




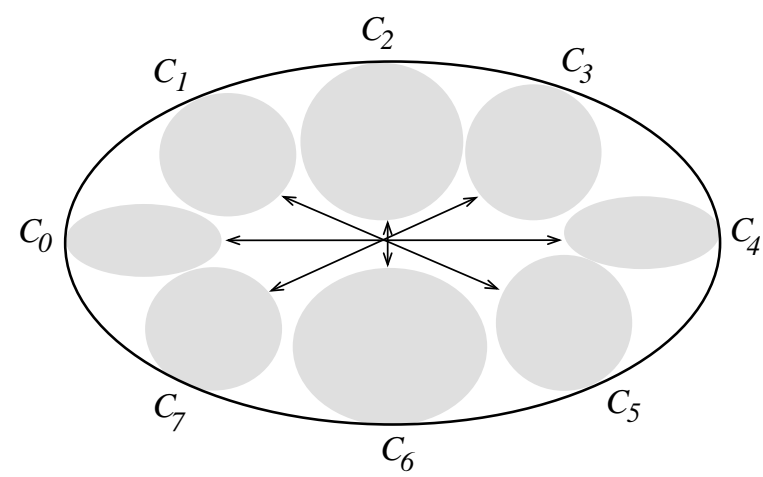

Figure 4: The point set partitioned into $2 k$ clusters.

$$
\begin{array}{ll}
D_{2}=\operatorname{Diam}\left(C_{2} \cup C_{3} \cup C_{4} \cup C_{5}\right) & D_{2}^{\prime}=\operatorname{Diam}\left(C_{6} \cup C_{7} \cup C_{0} \cup C_{1}\right) \\
D_{3}=\operatorname{Diam}\left(C_{3} \cup C_{4} \cup C_{5} \cup C_{6}\right) & D_{3}^{\prime}=\operatorname{Diam}\left(C_{7} \cup C_{0} \cup C_{1} \cup C_{2}\right) .
\end{array}
$$

\section{References}

[1] S. G. Akl and K. A. Lyons, Parallel Computational Geometry, Prentice Hall, Englewood Cliffs, New Jersey, 1993.

[2] T. Asano, B. K. Bhattacharya, J. M. Keil and F. F. Yao, "Clustering algorithms based on minimum and maximum spanning trees," Proc. of the Fourth Annual Symposium on Computational Geometry, 1988, 252-257.

[3] D. Avis, Diameter Partitioning, Discrete and Computational Geometry, 1, 1986, 265-276.

[4] C. Monma and S. Suri , "Partitioning points and graphs to minimize the maximum or the sum of diameters," Proceedings of the Sixth International Conference on the Theory and Applications of Graphs (Wiley, New York, 1989), 899-912. 